\title{
Numerical simulation of the de-bonding phenomenon of FRCM strengthening systems
}

\author{
Ernesto Grande \\ University Guglielmo Marconi, Department of Sustainability Engineering, via Plinio 44, 00193-Roma, Italy \\ e.grande@unimarconi.it, bttp:/ / orcid.org/0000-0002-3651-1975
}

Maura Imbimbo, Sonia Marfia

University of Cassino and Southern Lazio, Dep. of Civil and Mechanical Engineering, via G. Di Biasio 43, Cassino, Italy mimbimbo@unicas.it,http://orcid.org/0000-0003-3163-3073

marfia@unicas.it, bttp://orcid.org/0000-0002-2166-9788

\section{Elio Sacco}

University of Naples Federico II, Department of Structures in Engineering and Architecture, Via Claudio 21, Naples. Italy Elio.sacco@unina.it bttp:// orcid.org/0000-0002-3948-4781

\begin{abstract}
Aim of the paper is to present a one dimensional simple model for the study of the bond behavior of Fabric Reinforced Cementitious Matrix (FRCM) strengthening systems externally applied to structural substrates. The equilibrium of an infinitesimal portion of the reinforcement and the mortar layers composing the strengthening systems allows to derive the governing equations. An analytical solution is determined solving the system of differential equations. In particular, in the first part of the paper a nonlinear shear-stress slip law characterized by a brittle post-peak behavior with a residual shear strength in the post peak phase is introduced for either the lower reinforcement-mortar interface (approach 1) or both the lower and the upper interface (approach 2). In the latter approach, a calibration of the shear strength of the upper interface is proposed in order to implicitly account for the effect of the damage of the mortar on the bond behavior. In the second part of the paper it is presented the solution of the problem in the case of softening behavior by approximating the shear-stress slip law throughout a step function. Comparisons with experimental data, available in literature, are presented in order to assess the reliability of the proposed approach.
\end{abstract}

KEYwORDS. FRCM; De-bonding; Analytical model; Interface.

\section{OPEN ACCESS}

Citation: Grande, E., Imbimbo, M., Marfia, S., Sacco, E., Numerical simulation of the debonding phenomenon of FRCM strengthening systems, Frattura ed Integrità Strutturale, 47 (2019) 321-333.

Received: 23.10.2018

Accepted: 24.11.2018 Published: 01.01.2019

Copyright: (C) 2019 This is an open access article under the terms of the CC-BY 4.0, which permits unrestricted use, distribution, and reproduction in any medium, provided the original author and source are credited. 


\section{INTRODUCTION}

$\mathrm{T}$

he reinforcement of existing structures has always been a relevant problem both in the technical and scientific civil engineering community. Lately, the study and design of new reinforcement materials is a challenging issue. In particular, fabric reinforced cementitious matrix (FRCM) is an emerging strengthening system obtained embedding a grid of the carbon, glass or aramid reinforcement in an inorganic matrix. In general, the matrix is applied as a double layer incorporating the reinforcement. Nowadays, FRCM systems are used in the current practice to reinforce concrete and masonry structures.

Some experimental investigations [1-7] and theoretical/numerical studies ([2, 3, 8-16] on FRCM strengthening systems are available in the recent literature. They testify the efficacy and advantages of FRCM systems together with the need to investigate aspects specifically characterizing the bond behavior of this new family of strengthening systems.

The experimental investigations are mainly shear-lap tests that analyze the local bond behavior of FRCMs. From the experimental evidence different failure mechanisms can occur, such as a cohesive failure of the substrate, de-bonding at the reinforcement/substrate interface, de-bonding at the reinforcement/matrix interface, sliding of the reinforcement, tensile failure of the reinforcement in the un-bonded portion and tensile failure of the reinforcement within the mortar.

The above mechanism occurrence depends on the characteristics of the strengthening system as well as of the support, such as the mechanical properties of the materials, the thickness of the mortar layers and the configuration of the reinforcement. These mechanisms particularly underline the role of additional phenomena to be necessarily considered for the study and the development of theoretical models/design formulas specific for FRCMs.

Regarding the theoretical and numerical studies, the approaches available in literature show particular interest to the derivation of simple laws able to simulate the de-bonding phenomenon and, moreover, models able to account for additional phenomena specific of FRCMs.

In [2] the shear stress-slip law at the interface level was obtained throughout a procedure applied to steel and carbon FRCM strengthening systems externally embedded on masonry supports. The procedure was carried out by directly considering the experimental data and in particular the strain gauge measurements. A procedure for the derivation of the shear stress-slip law for FRCMs based on the experimental data was also proposed in [3]. In particular, the authors firstly estimated the fracture energy by using the well-known formula derived by the theory of linear fracture mechanics and, subsequently, they performed numerical FE analyses to detect the optimal values of the parameters of the cohesive shear stress-slip law.

In[4] the bond behavior of FRCM-to-concrete was analytically examined by using a general approach applied to the case of FRP-materials. The results emerged from this study particularly emphasized the role of the pronounced descending branch of the calibrated laws in leading to large values of the effective anchorage length. In addition, lower values of bond shear stresses on the concrete surface with respect to those typically characterizing FRP strengthening systems were observed. A similar approach was also presented in [3] for the case of carbon-FRCM materials externally applied on masonry supports.

In [13] two approaches for numerically studying the bond behavior of masonry specimens strengthened with FRCMs were proposed. The first one, consisted of an analytical-numerical approach specifically accounting for the interaction between the reinforcement and the mortar; the second approach consisted of a full 3D-FEM non-linear approach obtained as an extension of the procedure originally adopted in [13] and in [15].

A recent study presented in $[9,16]$ was mainly devoted to investigate the influence of the upper mortar layer on the bond behavior of FRCM-strengthening systems applied on structural supports. In particular, the authors carried out a theoretical modeling approach based on the solution of a system of differential equations obtained by introducing equilibrium considerations. From the study emerged interesting aspects concerning the role of the upper mortar layer on the debonding process of FRCMs. Among these, it was observed that increasing the applied load after the occurrence of the de-bonding between the reinforcement and the upper interface it does not lead to further increases of the peak value of normal stresses of the upper mortar. On the other hand, after the occurrence of the first crack at the upper mortar, only the peak value of slips at the lower interface continues to increase whilst the peak value of slips at the upper interface does not significantly increase.

In [10] it was proposed a simple approach for the study of the bond behavior of FRCM applied to concrete supports able to enable the use of a common interface modeling strategy by implicitly introducing the effect of the damage of the matrix into the shear behavior of the reinforcement/mortar interface layer.

In this paper a one dimensional simple model, based on the one presented in [9] and in [16], is proposed for the study of the bond behavior of FRCM strengthening systems externally applied to masonry substrates. The model is mainly 
characterized by the derivation of the explicit solution of a system of differential equations obtained by considering the equilibrium of an infinitesimal portion of the reinforcement and the mortar layers composing the strengthening systems. In order to model the slip between the reinforcement and the upper and lower mortar layers, two approaches are considered. The first approach (denoted in the following approach 1), considers a nonlinear behavior of the lower reinforcement/mortar interface only, by considering a shear stress-slip constitutive law characterized by a linear brittle behavior with a residual strength in the post-peak phase. On the other hand, the approach 2 assumes a nonlinear behavior for both the lower and the upper reinforcement/mortar interface, still considering a shear stress-slip constitutive law characterized by a linear fragile behavior with a residual strength in the post-peak phase. Moreover, in the latter approach, a calibration of the shear strength of the upper interface is proposed in order to implicitly account for the effect of the damage of the mortar on the contribution of this component of the strengthening system.

In addition to these approaches, in the second part of the paper is presented the analytical solution in case of a shear stress-slip law characterized by a linear softening behavior in the post-peak phase. In particular, a step function approximating the law together with the procedure carried out from the approach 2 are used in order to derive the analytical solution.

The proposed approaches are validated in the paper by considering experimental results derived from the literature. Moreover, the results are also compared with the ones obtained by the model recently proposed by [9,16], where, differently from the proposed approaches, the damage of the upper mortar was explicitly introduced in the model by assuming a nonlinear behavior in terms of normal stress-strain for the upper mortar layer. Although this assumption allows to account for the phenomena generally observed, it leads to a computational effort significantly greater than the one characterized the two approaches proposed in this paper.

\section{ACCOUNTED MODEL AND APPROACHES}

he model here considered for the study of the bond behavior of FRCM systems externally applied on masonry or concrete supports is based on the work in [9, 16]. Indeed, considering the scheme shown in Fig. 1, the main components characterizing the model are: a cohesive support, a lower mortar layer, a lower interface, the strengthening, an upper interface and an upper mortar layer. Introducing a reference axis $x$ in the direction of the reinforcement system and fixing the origin in correspondence of the unloaded section, the equilibrium of forces characterizing an infinitesimal portion of the reinforcement and the upper mortar layer (see Fig. 1) leads to the following system of differential equations governing the problem of the bond behavior:

$$
\left\{\begin{array}{l}
\frac{d \sigma_{p}}{d x} b_{p} t_{p}-\left[\tau^{e}\left(s^{e}\right)+\tau^{i}\left(s^{i}\right)\right] b_{p}=0 \\
\frac{d \sigma_{c}^{e}}{d x} b_{p} t_{c}^{e}+\tau^{e}\left(s^{e}\right) b_{p}=0
\end{array}\right.
$$

where $\sigma_{p}$ and $\sigma_{c}^{e}$ are the normal stresses in the reinforcement and in the upper mortar, respectively; $t_{p}$ and $t_{c}^{e}$ are the thicknesses of the reinforcement and the upper mortar, respectively; $\tau^{i}$ and $\tau^{e}$ are the shear stresses at lower and upper interfaces, respectively, both depending on the corresponding slips $s^{i}$ and $s^{e} ; b_{p}$ is the width of the reinforcement.

Introducing the following hypotheses:

- the support and the lower mortar layer are assumed to be rigid;

- the (lower and upper) mortar/reinforcement interfaces are modeled as zero-thickness elements with only shear deformability;

- the upper mortar layer and the reinforcement are assumed deformable only axially.

it is possible to write the displacements of both the reinforcement and the upper mortar layer (namely $u_{p}$ and $u_{c}^{e}$, respectively) as functions of the slip of the lower and upper interfaces:

$$
\begin{aligned}
& u_{p}=s^{i} \\
& u_{c}^{e}=s^{i}-s^{e}
\end{aligned}
$$




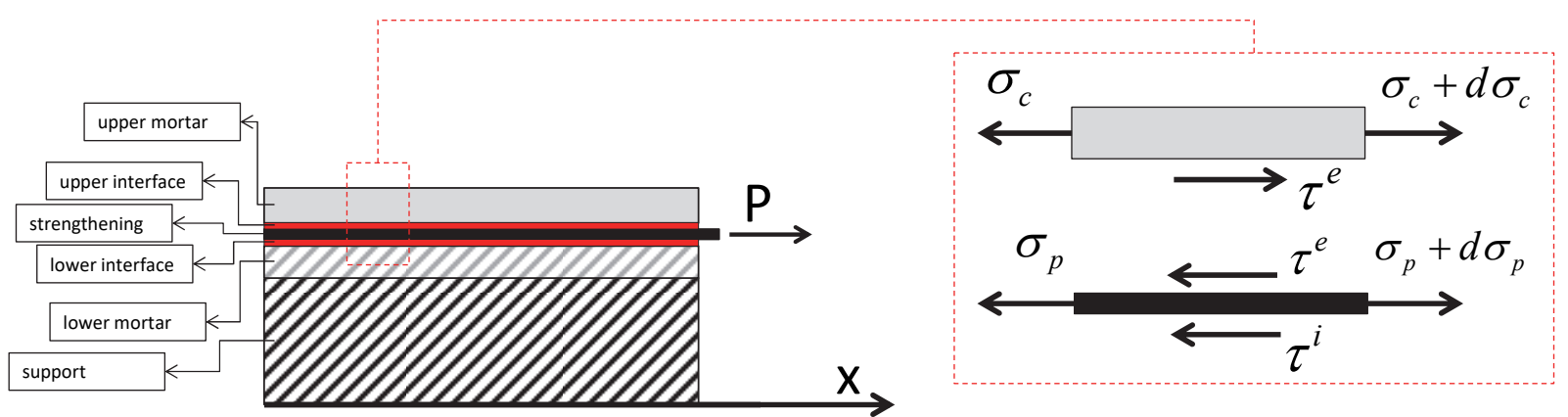

Figure 1: Schematic of an infinitesimal portion of the strengthening system and the upper mortar component used for performing the equilibrium of the involved forces.

Considering a linear-elastic behavior for both the reinforcement and the mortar:

$$
\begin{aligned}
& \sigma_{p}=E_{p} \frac{d u_{p}}{d x}=E_{p} \frac{d s^{i}}{d x} \\
& \sigma_{c}^{e}=E_{c} \frac{d u_{c}^{e}}{d x}=E_{c}\left(\frac{d s^{i}}{d x}-\frac{d s^{e}}{d x}\right)
\end{aligned}
$$

the system of differential Eqns. (1) becomes:

$$
\left\{\begin{array}{l}
\frac{d^{2} s^{i}}{d x^{2}}-K_{1}\left[\tau^{e}\left(s^{e}\right)+\tau^{i}\left(s^{i}\right)\right]=0 \\
\left(\frac{d^{2} s^{i}}{d x^{2}}-\frac{d^{2} s^{e}}{d x^{2}}\right)+K_{2} \tau^{e}\left(s^{e}\right)=0
\end{array}\right.
$$

where $K_{1}$ and $K_{2}$ are two constants equal to:

$$
K_{1}=\frac{1}{E_{p} t_{p}}, K_{2}=\frac{1}{E_{c} t_{c}^{e}}
$$

Considering the system (4), the explicit solution is here derived by introducing different shear stress-slip laws characterizing the behavior of the reinforcement/mortar interface.

\section{Approach 1: nonlinear behavior of the lower interface}

A preliminary approach is based on the assumption of a linear-fragile behavior with a residual shear strength in the postpeak stage only for the lower interface:

$$
\begin{cases}\tau^{i}\left(s^{i}\right)=G^{i} s^{i} & s^{i} \leq s_{1} \\ \tau^{i}\left(s^{i}\right)=\tau_{r e s}^{i} & \text { otherwise }\end{cases}
$$

where $\tau_{\text {res }}^{i}$ is the residual value of the shear strength in the post-peak stage, and $G^{i}$ is the shear stiffness of the lower interface in the pre-peak stage.

Differently, a linear-elastic behavior is assumed for the upper interface $\tau^{e}\left(s^{e}\right)=G^{e} s^{e}$, where $G^{e}$ is the shear stiffness of the upper interface. 
On the basis of these assumptions it is evident that, after the attainment of the slip threshold value at the lower interface, two different parts characterize the behavior of the specimen: part " 1 " where the upper mortar and the interfaces are both in the pre-peak stage and part " 2 " where the upper mortar and the upper interface are both in the pre-peak stage while the lower interface is de-bonded for a length $a$, representing an unknown of the problem. Consequently, four differential equations govern the problem.

The first two equations are derived by considering the equilibrium involving an infinitesimal portion of the strengthening system in the part " 1 ":

$$
\left\{\begin{array}{l}
\frac{d^{2} s_{1}^{i}}{d x^{2}}-K_{3}\left[s_{1}^{e}+\alpha s_{1}^{i}\right]=0 \\
\left(\frac{d^{2} s_{1}^{i}}{d x^{2}}-\frac{d^{2} s_{1}^{e}}{d x^{2}}\right)+K_{4} s_{1}^{e}=0
\end{array}\right.
$$

where: $K_{3}=K_{1} G^{e}, K_{4}=K_{2} G^{e}, \alpha=\frac{G^{i}}{G^{e}}$.

The other two equations are obtained through the equilibrium involving an infinitesimal portion of the strengthening system of the part "2":

$$
\left\{\begin{array}{l}
\frac{d^{2} s_{2}^{i}}{d x^{2}}-K_{3}\left[s_{2}^{e}+\beta\right]=0 \\
\left(\frac{d^{2} s_{2}^{i}}{d x^{2}}-\frac{d^{2} s_{2}^{e}}{d x^{2}}\right)+K_{4} s_{2}^{e}=0 \quad L-a<x<L
\end{array}\right.
$$

where $\beta=\frac{\tau_{\text {res }}^{i}}{G^{e}}$.

The system of differential Eqns. (7) and (8) has an analytical solution that depends on eight constants of integration determined by introducing suitable boundary conditions. In particular, the following conditions are indeed enforced:

$$
\begin{array}{ll}
\sigma_{P 1}(0)=0 & \\
\sigma_{c 1}^{e}(0)=0 & \sigma_{c 2}^{e}(L)=0 \\
\sigma_{c 1}^{e}(L-a)=\sigma_{c 2}^{e}(L-a) & \\
\sigma_{p 1}^{e}(L-a)=\sigma_{p 2}^{e}(L-a) & \\
s_{1}^{i}(L-a)=s_{2}^{i}(L-a) & s_{1}^{e}(L-a)=s_{2}^{e}(L-a) \\
s_{1}^{i}(L-a)=s_{1} &
\end{array}
$$

The solution is graphically reported in Fig. 2 by considering a length value of the part " 2 " equal to $a=50 \mathrm{~mm}$, a residual value of shear strength equal to zero and the data reported in Tab. 1.

\section{Approach 2: nonlinear behavior of both the interfaces}

As shown in $[11,17]$, the damage of the upper mortar generally occurs before the slipping of the reinforcement/mortar interfaces by particularly influencing the shear stress transfer mechanism.

This phenomenon is here simple introduced by considering an elastic-fragile behavior also for the upper interface and assuming for this component of the strengthening system a bond strength equal to the shear stress corresponding to the attainment of the tensile strength of the upper mortar layer. In other words, the effect of the damage of the upper mortar is implicitly introduced into the behavior of the upper interface. 

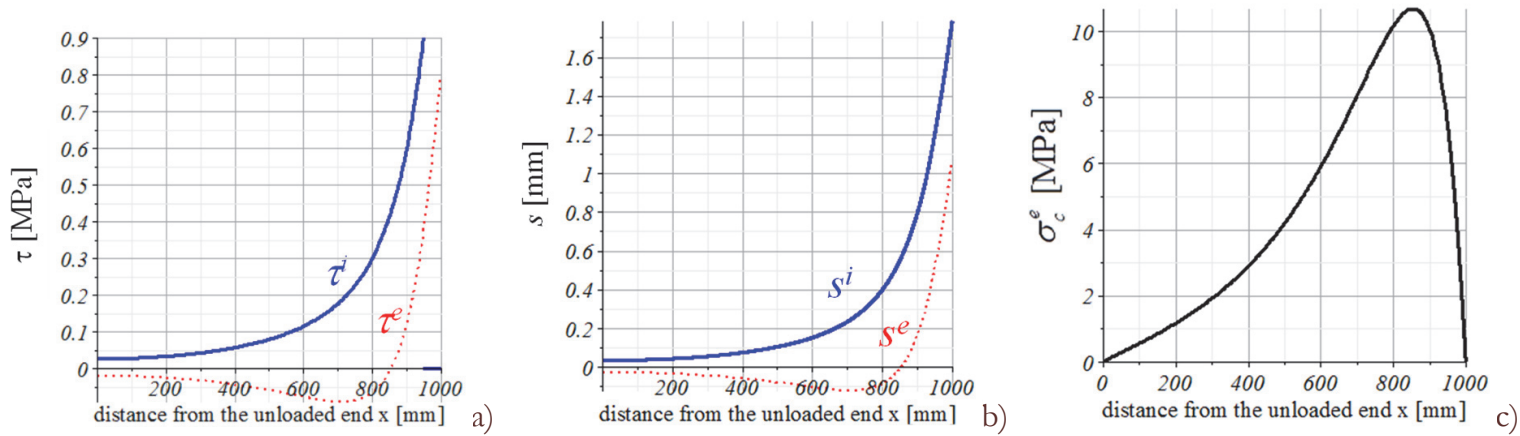

Figure 2: Approach 1: a) shear stress developing at the interfaces; b) slip of the interfaces; c) normal stresses at the upper mortar layer.

\begin{tabular}{ccc}
\hline $\begin{array}{c}\text { Young's modulus of the } \\
\text { reinforcement }\end{array}$ & symbol $[\mathrm{unit}]$ & value \\
$\begin{array}{c}\text { Young's modulus of the mortar } \\
\text { equivalent thickness of the }\end{array}$ & $\mathrm{E}_{\mathrm{p}}[\mathrm{MPa}]$ & 206000 \\
$\begin{array}{c}\text { reinforcement } \\
\text { thickness of the mortar }\end{array}$ & $\mathrm{t}_{\mathrm{p}}[\mathrm{mm}]$ & 7000 \\
width of the reinforcement & $\mathrm{t}_{\mathrm{c}}[\mathrm{mm}]$ & 0.054 \\
width of the mortar & $\mathrm{b}_{\mathrm{p}}[\mathrm{mm}]$ & 4 \\
bond length & $\mathrm{b}_{\mathrm{c}}[\mathrm{mm}]$ & 60 \\
\hline
\end{tabular}

Table 1: Data accounted for numerical analyses.

Considering this assumption, the system of equations governing the problem has to account for the development of three possible parts characterizing the behavior of the specimen: part " 1 ": $0<\mathrm{x}<\mathrm{L}-(\mathrm{a}+\mathrm{b})$, where both the interfaces are in the pre-peak stage and normal stresses in the upper mortar are lower than the tensile strength; part " 2 ": L- $(a+b)<x<L-b$, where the upper interface is in the post-peak stage (the length of this zone is equal to a); part " 3 ": L-b $<x<\mathrm{L}$, where both the interfaces are in the post-peak stage (the length of this zone is equal to b). In particular, while the equations characterizing the part " 1 " are the eqns. (7), the equations characterizing the part " 2 " are:

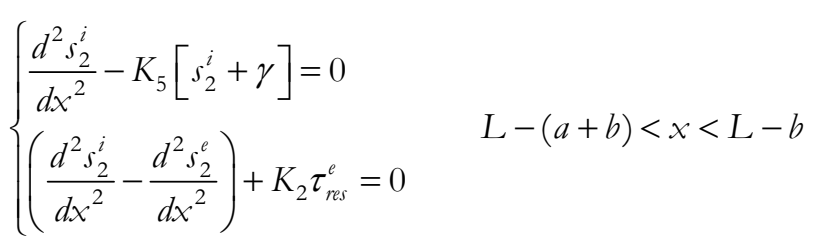

and the equations characterizing the part 3 are:

$$
\left\{\begin{array}{l}
\frac{d^{2} s_{3}^{i}}{d x^{2}}-K_{1}\left[\tau_{r e s}^{e}+\tau_{r e s}^{i}\right]=0 \\
\left(\frac{d^{2} s_{3}^{i}}{d x^{2}}-\frac{d^{2} s_{3}^{e}}{d x^{2}}\right)+K_{2} \tau_{r e s}^{e}=0
\end{array}\right.
$$

where: $\gamma=\frac{\tau_{r e s}^{e}}{G^{i}}, K_{5}=K_{1} G^{i}$, $a$ is the length of the part 2, $\mathrm{b}$ is the length of the part $3, \tau_{r e s}^{e}$ and $\tau_{r e s}^{i}$ are the residual shear strength values are of the upper and lower interfaces respectively.

The whole system of differential Eqns. (7), (10) and (11) has an analytical solution that depends on twelve constants of integration determined by introducing suitable boundary conditions similar to the ones introduced for the approach 1. The solution is graphically reported in Fig. 3 by considering a length value of the part " 2 " equal to a=50 mm, a length of 
the part " 3 " equal to $b=50 \mathrm{~mm}$, a shear strength of the lower mortar equal to $0.9 \mathrm{MPa}$, a shear strength of the upper mortar equal to $0.45 \mathrm{MPa}$, a residual value of shear strength equal to zero for both the interfaces and the data reported in Tab. 1.
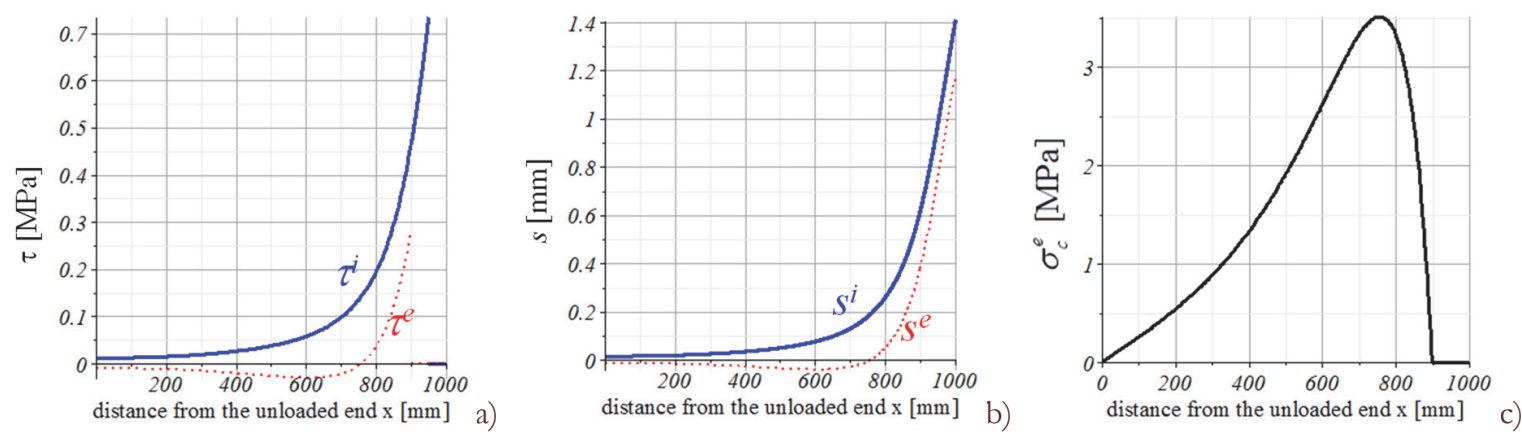

Figure 3: Approach 2: a) shear stress developing at the interfaces; b) slip of the interfaces; c) normal stresses at the upper mortar layer.

Numerical applications for the approach 1 and the approach 2

In order to assess the capability of the proposed model in providing a reliable prediction of the bond behavior of FRCM strengthening systems, some case studies derived from the current literature are considered ([4]). The case studies, here considered, consist of single lap shear tests of concrete blocks strengthened by a bidirectional unbalanced PBO fiber net with two mortar layers. In particular, in the present research the specimens are considered characterized by a bond length equal to $450 \mathrm{~mm}$ and two different values of the reinforcement width: $b_{p}=60 \mathrm{~mm}$ and $b_{p}=80$ mm. These tests are of particular relevance since the experimental outcomes showed the damage of the upper mortar layer of tested specimens before the slipping at the interface level.

Regarding the application of the approach 1, a shear stress-slip law for the lower interface characterized by a shear strength equal to $0.9 \mathrm{MPa}$ and a slip threshold equal to $1.2 \mathrm{~mm}$ is considered (see [4]). On the other hand, for the approach 2 a shear strength value of the upper interface corresponding to the attainment of the tensile strength of the upper mortar layer $\left(f_{c t}=3.5 \mathrm{MPa}\right)$ is considered. For both the approaches, a null value of the residual shear strength is assumed for both the interfaces.

The obtained results are shown in Fig. 4 in terms of applied load P versus the slip of the lower interface at the loaded section. In the same figure the envelop of the experimental curves (grey region) and the curve carried out in [4] are also reported.
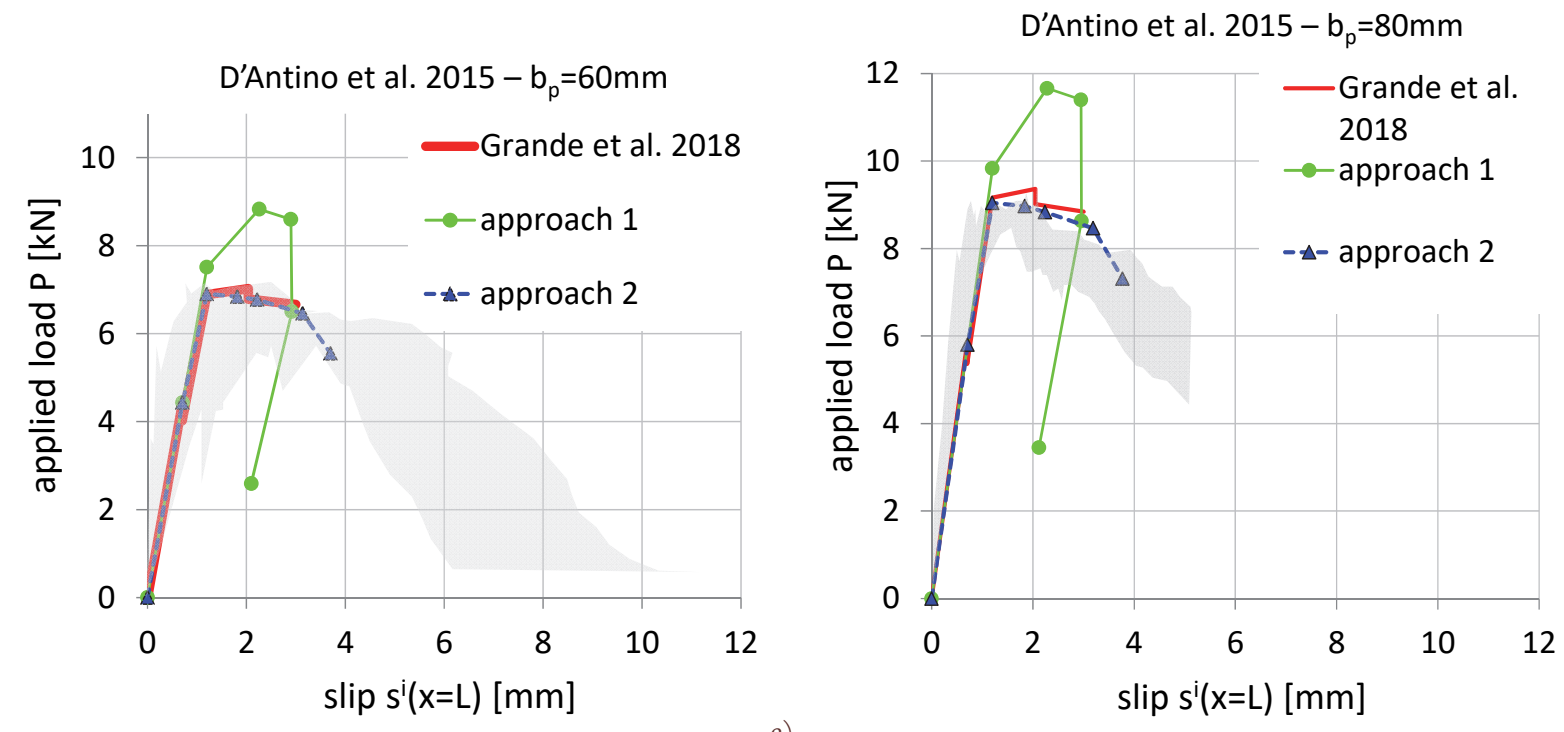

a)

Figure 4: Comparison with experimental tests in terms of applied force vs. slip curves. 
From the plots clearly emerges the importance of introducing in the model the influence of the damage of mortar on the contribution of the upper interface. Indeed, while the curves deduced from the approach 1 overestimate the experimental peak load, the curves derived by using the approach 2 provide a good approximation of the experimental outcomes.

\section{APPROXIMATE SOLUTION IN CASE OF SOFTENING BEHAVIOR}

I

$\mathrm{n}$ the previous section a solution of the de-bonding problem has been presented by introducing simplified shear stress-slip laws for the interfaces assumed with a perfect brittle post-peak behavior. This has allowed to obtain the analytical solution of the problem and to easily analyze different damage stages.

The majority of studies available in the current literature are instead based on the use of constitutive laws where the softening governs the post- peak phase of the interface (cohesive models). The use of this type of laws at the interface level generally does not lead to computational problems when a Finite Element approach, or other types of numerical techniques, are used. On the contrary, it can present some drawbacks for the derivation of an analytical solution, particularly in the case of FRCM systems where there are two interfaces interacting among them in the de-bonding process.

For this reason, the analytical solution is here derived by approximating the linear descending post-peak branch of the shear stress-slip law throughout a step function, i.e. a piecewise constant function (Fig. 5), and following the approach presented in the first part of the paper. Moreover, taking into account the 'approach 2' presented in the first part of the paper, the softening behavior is only introduced for the lower interface, whilst a linear-brittle behavior is accounted for the upper interface by opportunely calibrating the corresponding bond strength.

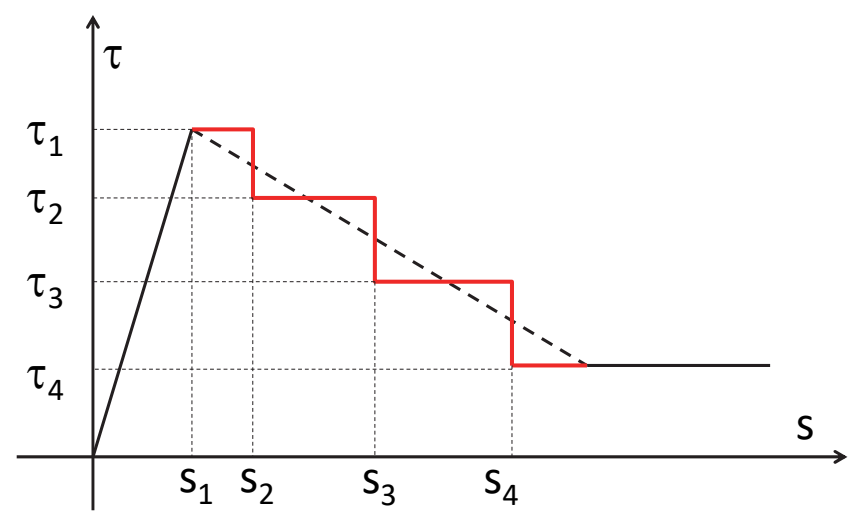

Figure 5: Scheme used for approximating the softening branch of the shear stress-slip law.

Also in this case, the system of equations governing the problem depends on the status of the two interfaces (see Fig. 6). Indeed, increasing the applied load $\mathrm{P}$, the first phase is certainly characterized by the pre-peak stage of both the interfaces (Fig. 6.a). In this case, the system of the two equations governing the problem is:

$$
\left\{\begin{array}{l}
\frac{d^{2} s^{i}}{d x^{2}}-K_{1}\left[\tau^{e}\left(s^{e}\right)+\tau^{i}\left(s^{i}\right)\right]=0 \\
\left(\frac{d^{2} s^{i}}{d x^{2}}-\frac{d^{2} s^{e}}{d x^{2}}\right)+K_{2} \tau^{e}\left(s^{e}\right)=0
\end{array}\right.
$$

where:

$$
\begin{aligned}
& \tau^{i}\left(s^{i}\right)=G^{i} s^{i} \\
& \tau^{e}\left(s^{e}\right)=G^{e} s^{e}
\end{aligned}
$$




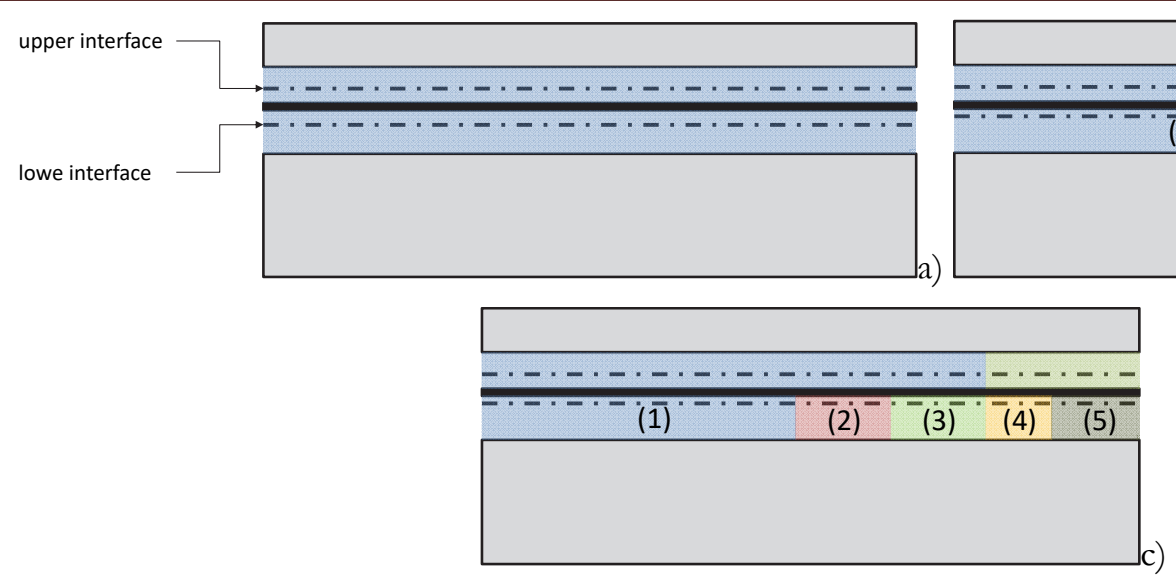

Figure 6: Scheme used for approximating the softening branch of the shear stress-slip law: a) phase $1 s^{i} \leq s_{1} ; s^{e} \leq s_{1}^{*}$; b) phase 2 zone 1: $s^{i} \leq s_{1} ; s^{e} \leq s_{1}^{*}$; zone 2 - zone $3: s^{i}>s_{1} ; s^{e} \leq s_{1}^{*}$; c) phase 3 zone $1: s^{i} \leq s_{1} ; s^{e} \leq s_{1}^{*}$; zone 2 - zone 3 : $s^{i}>s_{1} ; s^{e} \leq s_{1}^{*}$; zone $4-$ zone 5 : $s^{i}>s_{1} ; s^{e}>s_{1}^{*}$.

The analytical solution of this system depends on four constants of integration determined by introducing suitable boundary conditions. In particular, considering the attainment of the slip $s_{1}$ at the lower interface (generally, the lower interface exhibits higher values of shear stresses than the upper interface), the following conditions are indeed enforced:

$$
\begin{aligned}
& \sigma_{p}(0)=0 \\
& \sigma_{c}^{e}(0)=0 \\
& \sigma_{c}^{e}(L)=0 \\
& s^{i}(L)=s_{1}
\end{aligned}
$$

The subsequent phase is then characterized by the upper interface in the pre-peak stage and the lower interface in the post-peak stage (Fig. 6.b). This phase ends when the upper interface attains the slip $s_{1}$ at the loaded end. The occurrence of this condition identifies the number of steps approximating the descending branch of the shear stress-slip law of the lower interface involved in this phase. The number of equations governing the problem depends on the number of the zones in which the systems is divided and, thus, on the number of steps considered in the approximate descending branch of the interface constitutive law.

For instance, considering the case of the Fig. 6.b, it is supposed that there are three zones characterizing the behavior of the lower interface, which include two steps approximating the descending branch. Consequently, the differential equations composing the system are the following:

- zone 1:

$$
\left\{\begin{array}{l}
\frac{d^{2} s_{1}^{i}}{d x^{2}}-K_{1}\left[G^{e} s_{1}^{e}+G^{i} s_{1}^{i}\right]=0 \\
\left(\frac{d^{2} s_{1}^{i}}{d x^{2}}-\frac{d^{2} s_{1}^{e}}{d x^{2}}\right)+K_{2} G^{e} s_{1}^{e}=0
\end{array} \quad 0 \leq x<L-(a+b)\right.
$$

- zone 2:

$$
\left\{\begin{array}{l}
\frac{d^{2} s_{2}^{i}}{d x^{2}}-K_{1}\left[G^{e} s_{2}^{e}+\tau_{2}\right]=0 \\
\left(\frac{d^{2} s_{2}^{i}}{d x^{2}}-\frac{d^{2} s_{2}^{e}}{d x^{2}}\right)+K_{2} G^{e} s_{2}^{e}=0
\end{array} \quad L-(a+b) \leq x<L-b\right.
$$


- zone 3:

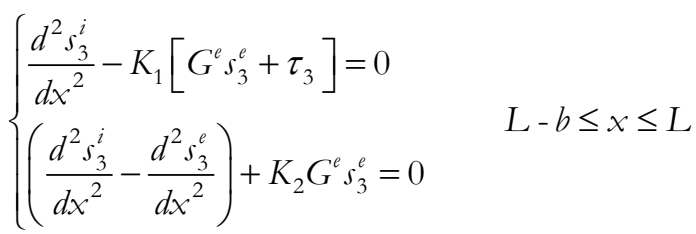

where $\tau_{2}$ and $\tau_{3}$ are the shear stress values identified by the steps approximating the descending branch in the phase 2 . In this case, it is necessary to introduce twelve boundary conditions. Four boundary conditions concern the two end sections of the specimen where it is stated that: the stress is zero at the free end of the reinforcement; the stresses are zero at two ends of the upper mortar layer; the upper interface attains the slip $s_{1}^{*}$ at the loaded end, i.e. the slip corresponding to the attainment of the tensile strength of the upper mortar layer, i.e.:

$$
\begin{gathered}
\sigma_{p, 1}(0)=0 \\
\sigma_{c, 1}^{e}(0)=0 \\
\sigma_{c, 3}^{e}(L)=0 \\
s_{3}^{e}(L)=s_{1}^{*}
\end{gathered}
$$

The other boundary conditions concern the continuity of normal stresses in the mortar layer and in the reinforcement, the continuity of the slip at the lower and upper layers at the section:

$$
\begin{array}{ll}
\sigma_{c 1}^{e}(L-(a+b))=\sigma_{c 2}^{e}(L-(a+b)) & \sigma_{p 1}^{e}(L-(a+b))=\sigma_{p 2}^{e}(L-(a+b)) \\
\sigma_{c 2}^{e}(L-b)=\sigma_{c 3}^{e}(L-b) & \sigma_{p 2}^{e}(L-b)=\sigma_{p 3}^{e}(L-b) \\
s_{1}^{i}(L-(a+b))=s_{2}^{i}(L-(a+b)) & s_{1}^{e}(L-(a+b))=s_{2}^{e}(L-(a+b)) \\
s_{1}^{i}(L-b)=s_{2}^{i}(L-b) & s_{1}^{e}(L-b)=s_{2}^{e}(L-b)
\end{array}
$$

Moreover, due to the introduction of the additional unknowns a and b, i.e. the length of the zone 2 and the zone 3 respectively, it is necessary to introduce the following two additional boundary conditions:

$$
\begin{aligned}
& s_{2}^{i}(L-b)=s_{2} \\
& s_{3}^{i}(L)=s_{3}
\end{aligned}
$$

where $s_{2}$ and $s_{3}$ are the slips identified by the two steps along the softening branch.

The subsequent phase (Fig. 6.c) is characterized by both the interfaces in the post-peak stage. An increase of the number of equations is still due to the steps discretizing the descending branch of the shear stress-slip law of the lower interface. Moreover, some of the zones are also characterized by a null value of the shear stress at the upper interface. Indeed, considering the example shown in Fig. 6.c, the set of equations characterized by this condition are the ones related to the last two zones:

- zone 4:

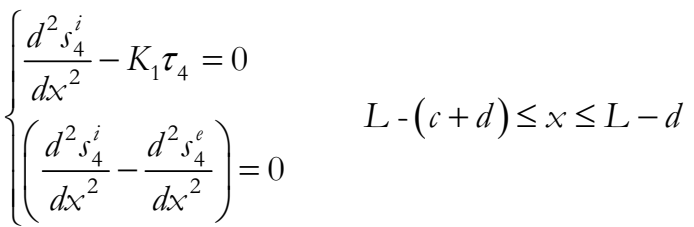


- zone 5:

$$
\left\{\begin{array}{l}
\frac{d^{2} s_{5}^{i}}{d x^{2}}-K_{1} \tau_{5}=0 \\
\left(\frac{d^{2} s_{5}^{i}}{d x^{2}}-\frac{d^{2} s_{5}^{e}}{d x^{2}}\right)=0
\end{array}\right.
$$

The boundary conditions are the same of those accounted for the previous phases and, also in this case, it is necessary to introduce additional boundary conditions for deriving the length of the different zones.

\section{Numerical applications for the case with softening}

Considering the approach based on the step function approximation, numerical applications are performed with reference to the case studies presented in the first part of the paper ([4]).

In particular, considering the shear stress-slip law proposed in [4], a bi-linear law has been derived (Fig. 7.a). Subsequently, this law has been approximated throughout a step function and assumed for the behavior of the lower interface (Fig. 7.b). Regarding the upper interface, a linear brittle shear stress-slip law has been considered by assuming a bond strength value equal to $0.9 \mathrm{MPa}$, which corresponds to the attainment of the tensile strength of the upper mortar layer (see the approach 2).
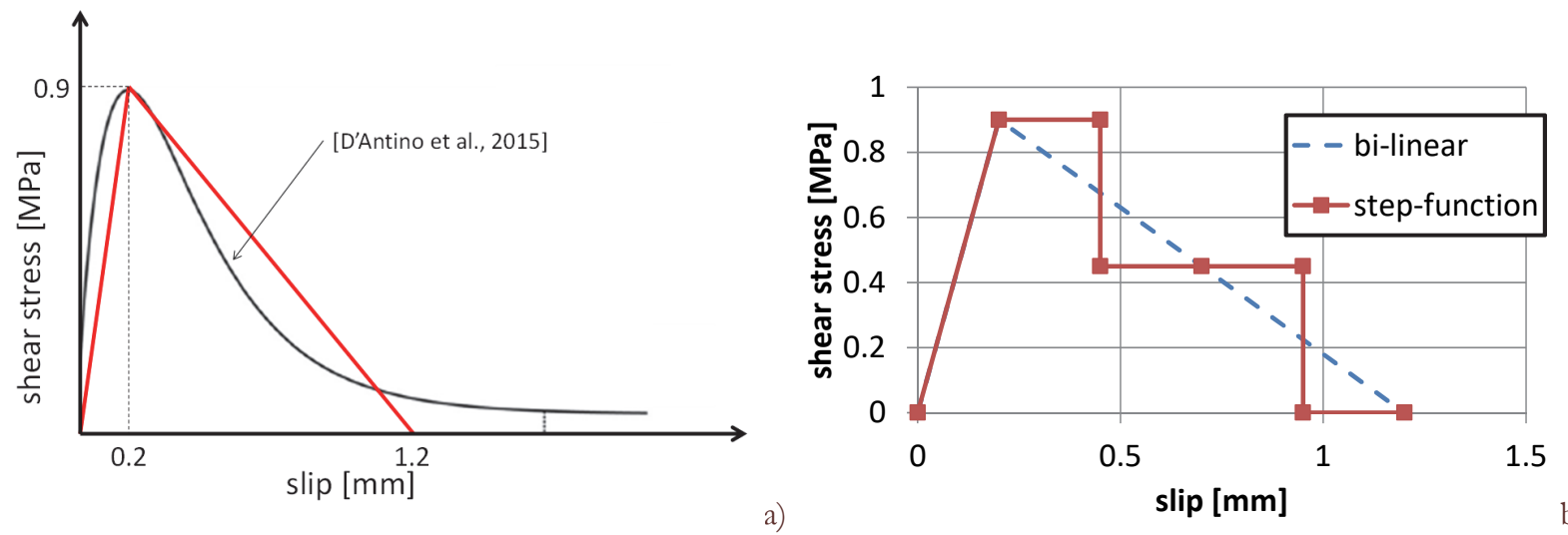

Figure 7: Shear stress-slip law accounted for the lower interface in the case of the approach based on the step function approximation.
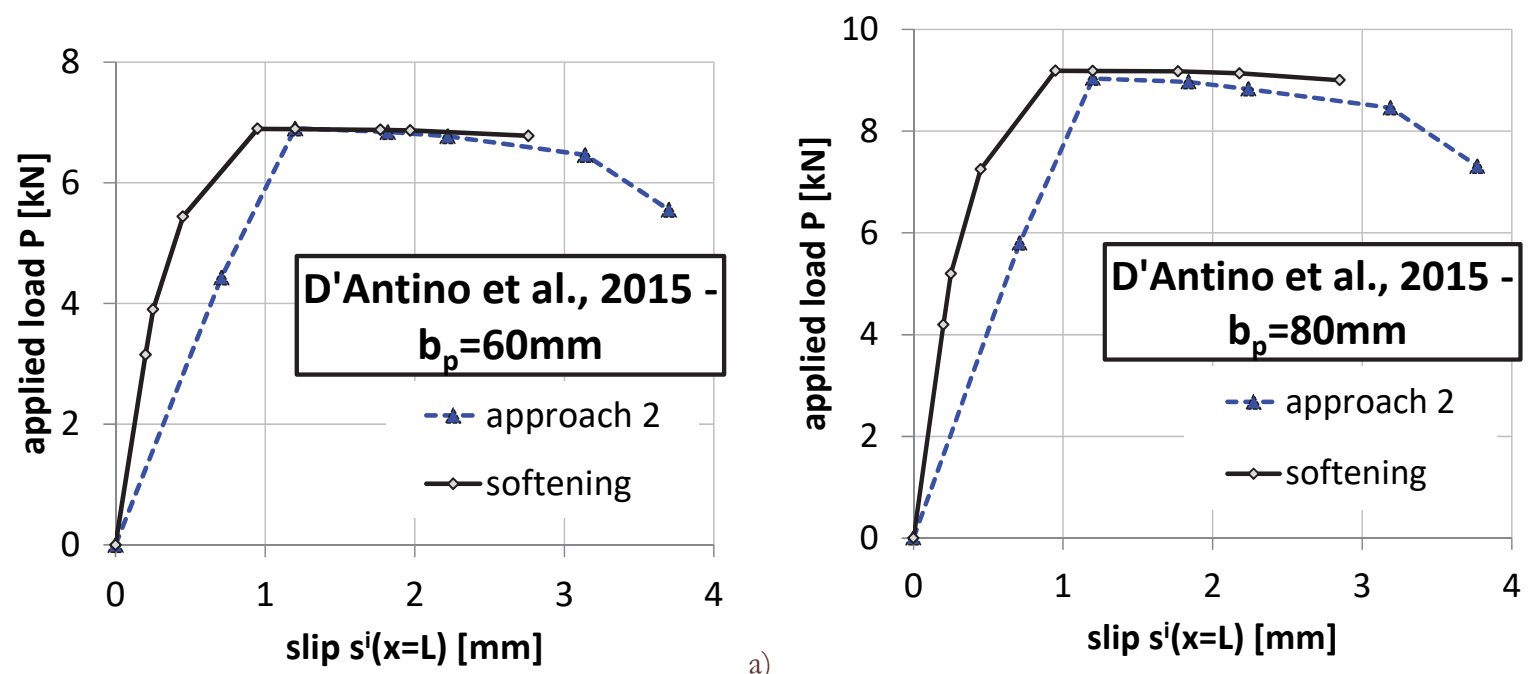

Figure 8: Results in terms of applied load vs. slip: comparison between the approach 2 and the case with softening. 
Then, considering the equations at the basis of the problem and the boundary conditions, both presented in the previous section, the analytical solution has been derived and presented in Fig. 8 in terms of applied load vs. the slip. In the same plot it is also reported the curve previously obtained by applying the approach 2 and then considering a linear-brittle behavior also for the lower interface. By examining the curves of the plot it emerges a similar value in terms of peak load and post-peak behavior. This outcome is due to the fact that the law assumed for the approach 2 and the one with softening are characterized by the same value of fracture energy: $\Gamma_{\mathrm{f}}=0.54 \mathrm{~N} / \mathrm{mm}$. On the other hand, the greater initial stiffness and the smoother post-peak branch of the case with softening are strictly related to the shape of the shear stressslip laws.

In Fig. 9, it is reported for the case with $b_{p}=60 \mathrm{~mm}$ the distributions along the bond length of shear stresses at the interfaces and normal stresses at the upper mortar layer for the load step corresponding to the attainment of the slip s1 at the lower interface. The plots underline the length of the four zones $\left(a_{1}=10 \mathrm{~mm} ; a_{2}=28.5 \mathrm{~mm} ; a_{3}=54 \mathrm{~mm} ; a_{4}=25 \mathrm{~mm}\right)$ characterizing the behavior of the lower interface. Moreover, it also emerges that: the maximum value of normal stresses in the upper mortar layer remains equal to the tensile strength because of the assumption of a reduced bond strength for the upper mortar; after the zone $\mathrm{a}_{1}$, since shear stresses in the upper interface are equal to zero, also the normal stresses at the upper mortar layer are zero.
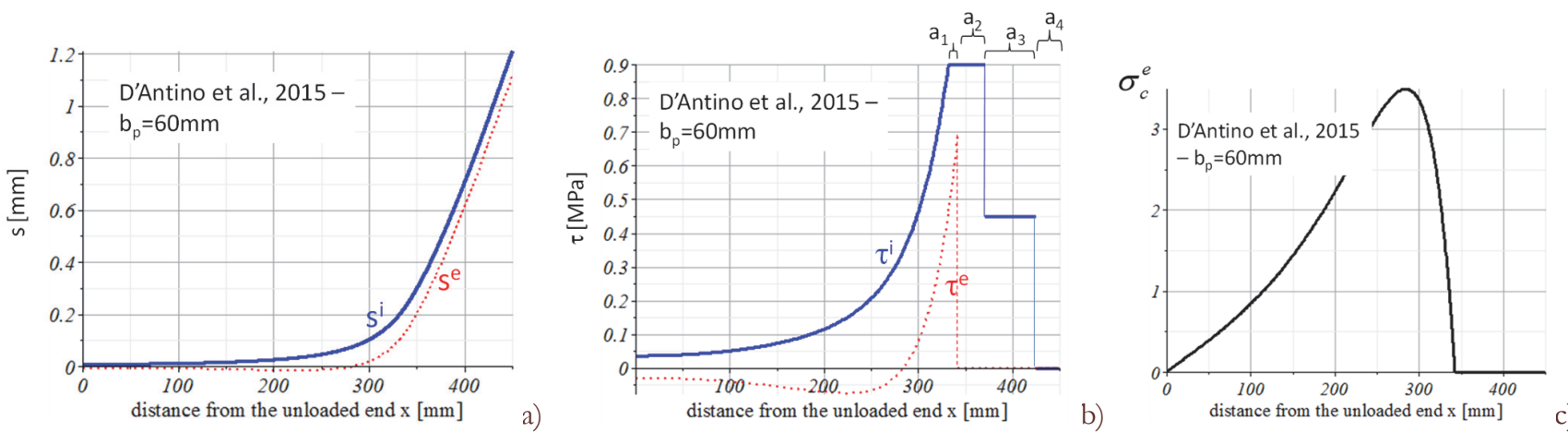

Figure 9: Results in terms of distribution along the bond length of a) slips, b) shear stresses, c) normal stresses at the step corresponding to the attainment of the slip value $\mathrm{si}=\mathrm{sf}=1.2 \mathrm{~mm}$ at the lower interface: case with softening.

\section{CONCLUSIVE REMARKS}

$\mathrm{I}$ $\mathrm{n}$ this paper a one-dimensional simplified model for studying the bond behavior of FRCM strengthening systems externally applied to masonry structures is proposed. The model is based on the study of an infinitesimal portion of the strengthening system composed by the reinforcement and the mortar layers, computing the explicit solution of a system of equilibrium differential equations. Interfaces are introduced between the reinforcement and the upper and lower mortar layers to model the possible slip phenomena. A nonlinear shear-stress slip laws, characterized by a brittle fracture with a residual strength in the post-peak stage, is adopted for the interfaces to derive the two approaches presented in the first part of the paper. These approaches differ only for the behavior of the upper interface: in the first one the upper interface is characterized by a linear behavior, while, in the second one by the proposed nonlinear response. Both the approaches have been applied to two case studies available in the literature.

From the results, approach 2 is able to better describe the experimental results, both in terms of peak load and post peak behavior, with respect to approach 1. In approach 2 the value of the peak shear stress in the upper interface is calibrated in order to take into account the damage occurring in the upper layer of mortar. The results are also compared with the ones obtained by the model proposed by $[9,16]$.

From a computational point of view, the presented model results simpler than the one proposed in [9,16], as it doesn't model directly the damage mechanism in the upper layer of mortar but it is able to take it into account by suitable setting the peak shear stress in the constitutive law of the upper interface.

In the second part of the paper it has been presented the analytical solution in the case of softening behavior of the lower interface. In particular, a step function has been introduced in order to approximate the softening branch. Also in this case the approach has been applied to the same case studies of literature. From the obtained results it is emerged a response similar to the one obtained in the approach 2 due to the important role played by the fracture energy, parameter common to the two models. 


\section{REFERENCES}

[1] D’Ambra, C., Lignola, G.P., Prota, A., Sacco, E., Fabbrocino, F. (2018). Experimental performance of FRCM retrofit on out-of-plane behaviour of clay brick walls, Compos. Part B Eng., 148, pp. 198-206.

DOI: 10.1016/j.compositesb.2018.04.062.

[2] D'Ambrisi, A., Feo, L., Focacci, F. (2012). Bond-slip relations for PBO-FRCM materials externally bonded to concrete, Compos. Part B Eng., 43(8), pp. 2938-2949. DOI: 10.1016/j.compositesb.2012.06.002.

[3] D’Ambrisi, A., Feo, L., Focacci, F. (2013). Experimental analysis on bond between PBO-FRCM strengthening materials and concrete, Compos. Part B Eng., 44, pp. 524-532. DOI: 10.1016/j.compositesb.2012.03.011.

[4] D'Antino, T., Sneed, L.H., Carloni, C., Pellegrino, C. (2015). Influence of the substrate characteristics on the bond behavior of PBO FRCM-concrete joints, Constr. Build. Mater., 101(1), pp. 838-850. DOI: 10.1016/j.conbuildmat.2015.10.045.

[5] de Felice, G., De Santis, S., Garmendia, L., Ghiassi, B., Larrinaga, P., Lourenço, P.B., Oliveira, D. V., Paolacci, F., Papanicolaou, C.G. (2014). Mortar-based systems for externally bonded strengthening of masonry, Mater. Struct. Constr., 47(2), pp. 2021-2037. DOI: 10.1617/s11527-014-0360-1.

[6] Grande, E., Imbimbo, M., Sacco, E. (2015). Investigation on the bond behavior of clay bricks reinforced with SRP and SRG strengthening systems, Mater. Struct. Constr., 48(11), pp. 3755-3770. DOI: 10.1617/s11527-014-0437-x.

[7] Marcari, G., Basili, M., Vestroni, F. (2017). Experimental investigation of tuff masonry panels reinforced with surface bonded basalt textile-reinforced mortar, Compos. Part B Eng., 108, pp. 131-142. DOI: 10.1016/j.compositesb.2016.09.094.

[8] Grande, E., Imbimbo, M., Sacco, E. (2013). Modeling and numerical analysis of the bond behavior of masonry elements strengthened with SRP/SRG, Compos. Part B Eng., 55, pp. 128-38.

[9] Grande, E., Imbimbo, M., Sacco, E. (2017). Local bond behavior of frcm strengthening systems: Some considerations about modeling and response, 747 KEM, pp. 101-107.

[10] Grande, E., Milani, G. (2018). Interface modeling approach for the study of the bond behavior of FRCM strengthening systems, Compos. Part B Eng., 141, pp. 221-233. DOI: 10.1016/j.compositesb.2017.12.052.

[11] Carbone, I. (2010). Delaminazione di compositi a matrice cementizia su supporti murari. Università degli Studi Roma Tre.

[12] Faella, C., Martinelli, E., Paciello, S., Perri, F. (2009).Composite materials for masonry structures: the adhesion issue. mechanics of masonry structures strengthened with composite materials, In: Proc. of 3rd Conf. on Mechanics of masonry structures strengthened with composite materials: modeling, testing, design, control - MuRiCo3, Venice (Italy), pp. 266-273.

[13] Carozzi, F.G., Milani, G., Poggi, C. (2014). Mechanical properties and numerical modeling of Fabric Reinforced Cementitious Matrix (FRCM) systems for strengthening of masonry structures, Compos. Struct., 107, pp. 711-725. DOI: 10.1016/j.compstruct.2013.08.026.

[14] Milani, G., Lourenço, P.B. (2012). 3D non-linear behavior of masonry arch bridges, Comput. Struct., 110, pp. 133150. DOI: $10.1016 /$ j.compstruc.2012.07.008.

[15] Milani, G., Tralli, A. (2012). A simple meso-macro model based on SQP for the non-linear analysis of masonry double curvature structures, Int. J. Solids Struct., 49(5), pp. 808-834. DOI: 10.1016/j.ijsolstr.2011.12.001.

[16] Grande, E., Imbimbo, M., Sacco, E. (2018). Numerical investigation on the bond behavior of FRCM strengthening systems, Compos. Part B Eng., 145, pp. 240-251. DOI: 10.1016/j.compositesb.2018.03.010. 